\title{
An audit of complications of complex operative gynaecological laparoscopy at a tertiary healthcare facility in South Africa
}

\author{
C N Omile, MB ChB; S Ramphal, MB ChB, FCOG (SA); J Moodley, MB ChB, FRCOG, FCOG (SA) \\ Department of Obstetrics and Gynaecology, School of Clinical Medicine, Nelson R Mandela School of Medicine, University of KwaZulu-Natal, \\ Durban, South Africa
}

Corresponding author: S Ramphal (RAMPHALS1@ukzn.ac.za)

Background. Operative laparoscopic surgery has many advantages over traditional/open laparotomy. However, it is also associated with complications particularly when performed for complex gynaecological procedures. There are very few reports on operative laparoscopic surgery from developing countries.

Objective. To evaluate the intra- and postoperative complications associated with laparoscopic surgery performed for complex gynaecological conditions by a single surgeon at a tertiary institution.

Method. This was a retrospective chart review of patients who underwent complex laparoscopic surgery between 2004 and 2016.

Results. We retrieved 446 patient records. Surgery for extensive endometriosis (29.8\%) and laparoscopic-assisted vaginal hysterectomy (29.8\%) were the most common procedures. Less than a tenth of patients $(4.3 \% ; n=19)$ had complications, 9 had minor injuries $(4$ inferior epigastric vessel injury, 1 peritoneal vessel injury, 1 omental vessel injury, 1 surface tissue bleeding and 2 cases of postoperative ileus) and 10 had major injuries ( 6 intestinal, 2 ureteric and 3 bladder). More than a quarter of complications $(26.3 \% ; n=5)$ occurred at the time of abdominal entry, $63.2 \%(n=12)$ occured intraoperatively and $10.5 \%(n=2)$ occurred postoperatively. All entry injuries were vascular. Intestinal injury was the most common intraoperative complication.

Conclusion. Despite the many advantages of laparoscopic surgery, complications occur particularly in patients with complex gynaecological pathology.

S Afr J Obstet Gynaecol 2020;26(3):115-120. https://doi/org/10.7196/SAJOG.2020.v26i3.2019

Operative laparoscopy is a common surgical intervention because it is less invasive and is associated with minimal scars, improved cosmesis, shorter hospital stay, less analgesia for postoperative pain and a rapid return to normal activity. ${ }^{[1]}$ In addition, it has better patient satisfaction compared with open surgery ${ }^{[1,2]}$

The laparoscopic approach is also useful in the management of patients who require re-exploration and correction of postoperative surgical complications irrespective of the initial surgical approach. ${ }^{[3]}$ However, complications and failure of laparoscopic surgical techniques with conversion to laparotomy do occur ${ }^{[3]}$ and the complication rates have been reported to increase when laparoscopic surgery is used for more complex pathology. ${ }^{[4]}$

An advanced gynaecological endoscopy unit was established at a new central hospital in Durban, South Africa (SA) in 2004. Patients from KwaZulu-Natal (KZN) Province with complex pathologies were referred to this unit. To date, there has been no audit of gynaecological laparoscopic procedures performed at this unit. Therefore, the aim of the present study was to determine the complication rates associated with advanced gynaecological laparoscopic surgery at this institution.

\section{Methods}

This was a retrospective study of patients who underwent complex operative gynaecological laparoscopy from 2004 - 2016. Regulatory permissions, both ethical and hospital authority (ref. no. BE463/17), were obtained to retrieve patient information from the hospital's electronic databases.

All patients were referrals from public and private healthcare facilities in KZN. The standard clinical practice included history taking, clinical examination, laboratory tests, and ultrasound and imaging investigations prior to the operation at the study site. Upon discharge from hospital, all patients were followed up for 1 month and patients who were stable were referred back to their local hospitals, while the rest were followed up at this institution for at least 3 months.

A pre-formatted data sheet was used to document all the relevant clinical and surgical information, which included demographic, intra- and postoperative data, and the detection and management of any complications. All laparoscopic procedures were performed by a single surgeon (SR).

Laparoscopy followed standard entry procedures. Most cases used the umbilicus as the primary site of the Veress needle entry for insufflation and primary trocar entry for laparoscopic vision. In selected cases such as adnexal surgery in pregnancy and previous midline laparotomy, the Palmer's point $(3 \mathrm{~cm}$ below the left coastal margin in the mid-clavicular line) was used.

Complications were classified as major or minor. Major complications included intraoperative complications such as visceral damage (bladder, intestine and ureter) and bleeding from major vessels, or detection of complications in the postoperative period 
that warranted re-laparoscopy or laparotomy. Minor complications included bleeding from omental and inferior epigastric vessels, and postoperative ileus.

Data were analysed using SPSS version 25 (IBM Corp., USA). Descriptive statistics such as frequencies and percentages were used to summarise categorical data.

\section{Results}

There were 446 cases of laparoscopic surgery during the 12-year period (2004 - 2016); 39\% $(n=174)$ of the surgeries were performed during $2004-2008,22.4 \%(n=100)$ were performed during 2009 - 2012, and 38.6\% ( $n=172)$ were performed during 2013 - 2016. The majority of the patients $(40 \% ; n=180)$ were in the 31 - 45 years age group while $16.4 \%(n=73)$ were $>60$ years. Less than a tenth of patients $(4.6 \% ; n=20)$ were underweight; $29.2 \%$ $(n=130)$ were obese and $4.9 \%(n=22)$ were morbidly obese with a body mass index (BMI) $>40 \mathrm{~kg} / \mathrm{m}^{2}$. More than a quarter of the patients $(26.5 \% ; n=118)$ had previous surgery, and $47.5 \%(n=56)$ of whom had a previous caesarean delivery. Moreover, 68 patients had previous laparoscopy with umbilical and abdominal scars in both iliac areas, $52.9 \%(n=36)$ for endometriosis, $23.5 \%(n=16)$ had laparoscopic-assisted vaginal hysterectomy (LAVH) and 11.8\% $(n=8)$ had adhesiolysis.

\section{Types of laparoscopic surgery}

The most common procedures performed were surgery for severe endometriosis (29.8\%; $n=133$ ) and LAVH (29.8\%; $n=133)$. More than a tenth of patients $(15.2 \%$; $n=68)$ with utero-vaginal prolapse had LAVH with uterosacral vault suspension. Laparoscopy and extensive adhesiolysis were conducted in patients $(8.9 \% ; n=39)$ for chronic pelvic pain. Other surgical procedures are shown in Table 1.

\section{Types of laparoscopic entry}

All patients except one had a Veress needle abdominal entry technique. A direct trocar entry was conducted for an extra-uterine

\begin{tabular}{ll} 
Table 1. Types of surgical procedures & \\
\hline Type of procedure & $\boldsymbol{n}(\%)$ \\
\hline Extensive adhesiolysis for unexplained pelvic pain & $39(8.9)$ \\
Total laparoscopic hysterectomy & $11(2.6)$ \\
$\begin{array}{l}\text { Laparoscopic removal of lost intrauterine contraceptive } \\
\text { device }\end{array}$ & $7(1.6)$ \\
Laparoscopic surgery for extra-uterine pregnancy in & $11(2.6)$ \\
second trimester & \\
Laparoscopic Burch Colpo-suspension & $5(1.2)$ \\
Laparoscopic myomectomy & $8(1.8)$ \\
Laparoscopically assisted vaginal hysterectomy & \\
Without uterosacral vault suspension & $65(14.6)$ \\
With uterosacral vault suspension & $68(15.2)$ \\
Laparoscopy for advanced stage endometriosis & $133(29.8)$ \\
Laparoscopic adnexal surgery in mid trimester of & $16(3.6)$ \\
pregnancy & \\
Laparoscopic urogenital fistulae repair & $16(3.6)$ \\
Laparoscopic Vecchietti procedure & $11(2.5)$ \\
Laparoscopic sacrocolpopexy & $36(8.1)$ \\
Laparoscopic uterosacral vault suspension & $3(0.7)$ \\
Laparoscopic radical hysterectomy for cervical cancer & $6(1.4)$ \\
Other procedures & $8(1.8)$ \\
&
\end{tabular}

pregnancy. The Veress needle and primary trocar entry at the umbilicus were used in $83.6 \%$ ( $n=373)$ of patients while the Palmer's point entry was utilised in 73 patients.

\section{Entry and intraoperative complications}

There were $3.8 \%(n=17)$ cases of direct surgery-related complications and $0.4 \%(n=2)$ cases of paralytic ileus, giving a total complication rate of $4.2 \%$ (Table 2 ).

The majority of complications occurred during the $2004-2008$ and 2013 - 2016 periods (Table 3 ).

Less than a quarter of complication $(23.5 \% ; n=4)$ were entry-related vascular injuries involving the inferior epigastric vessels during the secondary port insertion. There were no Veress needle related injuries. Two of these patients had a BMI between 25 and $29.9 \mathrm{~kg} / \mathrm{m}^{2}$, and the other two had a BMI of $30-34.9 \mathrm{~kg} / \mathrm{m}^{2}$. There was one primary trocar injury which was vascular in nature and identified within 24 hours following the surgery. This patient had surgery for severe endometriosis and was found to be clinically pale with a tachycardia 6 hours following the operation. A re-laparoscopy was performed and a bleeding umbilical peritoneal vessel at the primary port site was identified and coagulated.

There were 12 intraoperative surgery-related injuries. All occurred during the dissection phase that involved scissors. Less than a quarter of injuries $(17.0 \% ; n=2)$ were vascular, $50.0 \%(n=6)$ were intestinal and $33.0 \%(n=4)$ were urological. More than twothirds of the injuries $(66.7 \% ; n=8)$ were repaired laparoscopically and $25 \%(n=3)$ required immediate conversion to laparotomy for correction. In one patient with a ureteric injury, laparotomy was performed 18 days after initial surgery. Surgery for severe endometriosis had the most complications, occurring in five patients, followed by laparoscopic sacrocolpopexy (LSCP) and extensive adhesiolysis occurring in three and two patients, respectively (Table 4 ).

Specific to the intraoperative vascular injuries, one patient had bleeding from the omental vessels during extensive adhesiolysis and there was surface bleeding from a pedunculated fibroid in a patient undergoing ovarian cystectomy in the mid-trimester of pregnancy. Both were repaired laparoscopically with electro-cautery or suturing and none required blood transfusion.

Intraoperative injury to the small bowel occurred in two patients, the rectum in one case, and the sigmoid colon in three cases. In one case, small bowel injury occurred during extensive adhesiolysis, and this was repaired laparoscopically. In the other case, a small bowel injury occurred during separation of bowel from the uterus prior to LAVH. The hysterectomy was completed laparoscopically and a mini-laparotomy was performed and the bowel injury repaired. A rectal injury occurred during surgery for severe endometriosis with conversion to laparotomy for the repair. Injury to the sigmoid colon occurred in two patients during surgery for severe endometriosis and in one patient during LSCP. Repairs were performed laparoscopically in two patients and conversion to laparotomy for repair was carried out in one patient who had surgery for severe endometriosis.

Only four patients had urological injuries. There were two bladder injuries which occurred during LSCP and both were identified and repaired intraoperatively. Two ureteric injuries occurred in patients who had surgery for severe endometriosis. One of the ureteric injuries was discovered intraoperatively and repaired laparoscopically with the 


\section{RESEARCH}

aid of cystoscopy and stenting of the ureters. The second patient with ureteric injury presented 18 days following surgery with non-specific symptoms and deranged renal function. The patient had retrograde ureteric imaging in theatre and a left ureteric injury was identified. A laparotomy with ureteric re-implantation was performed. Table 5 describes all complications and management.

Table 2. Intraoperative and postoperative complications

\begin{tabular}{|c|c|c|c|c|c|c|c|c|c|}
\hline \multirow[b]{2}{*}{ Type } & & & \multicolumn{2}{|c|}{ Vascular, $n(\%)$} & \multicolumn{3}{|c|}{ Intestinal, $n(\%)$} & \multicolumn{2}{|c|}{ Urological, $n(\%)$} \\
\hline & & & Minor & Major & Small & Large & Rectum & Bladder & Ureter \\
\hline \multirow[t]{4}{*}{ Entry } & \multirow{2}{*}{$\begin{array}{l}\text { Primary } \\
\text { port }\end{array}$} & Immediate & - & - & - & - & - & - & - \\
\hline & & Within $24 \mathrm{~h}$ & 1 & - & & & & & \\
\hline & \multirow{2}{*}{$\begin{array}{l}\text { Secondary } \\
\text { port }\end{array}$} & Immediate & 4 & - & - & - & - & - & - \\
\hline & & Within $24 \mathrm{~h}$ & - & - & & & & & \\
\hline \multicolumn{3}{|l|}{ Total } & $5(1.1)$ & & & & & & \\
\hline \multirow[t]{3}{*}{ Surgery-related } & \multicolumn{2}{|c|}{ Intraoperative } & 2 & - & 2 & 3 & 1 & 2 & 1 \\
\hline & \multicolumn{2}{|c|}{ Within $24 \mathrm{~h}$} & - & - & - & - & - & - & - \\
\hline & \multicolumn{2}{|c|}{ After 1 week } & & - & - & - & - & - & 1 \\
\hline \multicolumn{3}{|l|}{ Sub-total } & 2 & & 6 & & & 4 & \\
\hline \multicolumn{3}{|l|}{ Total } & $12(2.7)$ & & & & & & \\
\hline \multicolumn{3}{|c|}{ Postoperative paralytic ileus } & $2(0.4)$ & & & & & & \\
\hline \multicolumn{3}{|l|}{ Total } & $2(0.4)$ & & & & & & \\
\hline \multicolumn{3}{|l|}{ Grand total } & $19(4.2)$ & & & & & & \\
\hline
\end{tabular}

Table 3. Laparoscopic surgical complications over the study period

\begin{tabular}{lllllll}
\hline & & \multicolumn{3}{c}{ Vascular } & & \\
\cline { 3 - 6 } & Procedures, $\boldsymbol{n}$ & Entry, $\boldsymbol{n}$ & Intraop., $\boldsymbol{n}$ & Intestinal, $\boldsymbol{n}$ & Urological, $\boldsymbol{n}$ & Postop. ileus, $\boldsymbol{n}$ \\
\hline $2004-2008$ & 174 & 2 & 1 & 2 & 2 & 1 \\
$2009-2012$ & 100 & 0 & & 1 & 0 & 0 \\
$2013-2016$ & 172 & 3 & 1 & 3 & 2 & 1 \\
Total & 446 & 7 & & & & \\
Intraop. $=$ intraoperative; postop. = postoperative.
\end{tabular}

Table 4. Procedures associated with intraoperative injury

\begin{tabular}{|c|c|c|c|c|c|c|c|c|}
\hline Procedures & $N$ & $\begin{array}{l}\text { Vascular, } \\
n\end{array}$ & $\begin{array}{l}\text { Bladder, } \\
n\end{array}$ & $\begin{array}{l}\text { Ureter, } \\
n\end{array}$ & $\begin{array}{l}\text { Sigmoid } \\
\text { colon, } \\
n\end{array}$ & $\begin{array}{l}\text { Small } \\
\text { intestine, } \\
n\end{array}$ & $\begin{array}{l}\text { Rectum, } \\
n\end{array}$ & $\begin{array}{l}\text { Total, } \\
n\end{array}$ \\
\hline Surgery for severe endometriosis & 133 & - & - & 2 & 2 & - & 1 & 5 \\
\hline LSCP & 36 & & 2 & & 1 & - & - & 3 \\
\hline Extensive adhesiolysis & 39 & 1 & - & - & - & 1 & - & 2 \\
\hline Surgery for adnexal mass in pregnancy & 16 & 1 & - & - & - & - & - & 1 \\
\hline LAVH & 133 & - & - & - & - & 1 & - & 1 \\
\hline
\end{tabular}

Table 5. Summary of complications and management

\begin{tabular}{|c|c|c|c|c|}
\hline \multicolumn{2}{|c|}{ Type of complication } & \multirow{2}{*}{$\begin{array}{l}\text { Patients, } \boldsymbol{n} \\
1\end{array}$} & \multirow{2}{*}{$\begin{array}{l}\text { Recognition time } \\
\text { Immediate }\end{array}$} & \multirow{2}{*}{$\begin{array}{l}\text { Management } \\
\text { Cautery }\end{array}$} \\
\hline Bleeding & Pedunculated fibroid & & & \\
\hline & Inferior epigastric & 4 & Immediate & Suture /cautery \\
\hline & Omentum & 1 & Immediate & Cautery \\
\hline & Post op-peritoneal at port site & 1 & within $24 \mathrm{~h}$ & Re-laparoscopy + suture \\
\hline \multirow[t]{2}{*}{ Intestinal } & Small intestine & 2 & Immediate & 1 converted to laparotomy, 1 laparoscopic repair \\
\hline & Sigmoid colon & 3 & Immediate & 1 converted to laparotomy, 2 laparoscopic repair \\
\hline \multirow[t]{3}{*}{ Urological } & Bladder & 2 & Immediate & Laparoscopic repair \\
\hline & Ureter & 1 & Immediate & Cystoscopy/stenting \\
\hline & Ureter & 1 & Delayed 14 days & Laparotomy \\
\hline Paralytic ile & & 2 & Postoperative & Conservative management \\
\hline
\end{tabular}


Table 6. Conversion to laparotomy

\begin{tabular}{ll}
\hline Reason for conversion & $\boldsymbol{n}$ (\%) \\
\hline Technical difficulty & $5(45.6)$ \\
Equipment failure & $1(9.0)$ \\
Absent device & $1(9.0)$ \\
Suspected bleeding & $1(9.0)$ \\
Viscus injury & $3(27.4)$ \\
Total & $11(100)$
\end{tabular}

\section{Conversion to laparotomy}

Less than a tenth of patients $(2.5 \% ; n=11)$ were converted to laparotomy (Table 6). Three were due to intraoperative injuries noticed during the procedure. Five cases were converted to laparotomy due to technical difficulty in dealing with excessive adhesions. Conversion occurred due to equipment failure and absent device in one patient. The eleventh case was a patient with suspected intraoperative bleeding; however, no active bleeding was identified.

\section{Postoperative complications}

Less than one percent of patients $(0.4 \% ; n=2)$ had postoperative ileus after LAVH and were managed conservatively. Both patients had abdominal discomfort and absent bowel sound post surgery. These patients improved with nasogastric suction, and recovered uneventfully (Tables 2 and 5).

\section{Follow-up}

All patients were seen at least 1 month postoperatively and no complications were noted.

\section{Discussion}

Of the 446 patients in the present study, $4.3 \% \quad(n=19)$ had complications. More than half of the patients $(52.6 \% ; n=10)$ had a major complication involving viscera, giving an incidence of $2.2 \%$. This complication rate is comparable with a rate of $2.0 \%$ reported by Shastri et al. ${ }^{[5]}$ and $1.9 \%$ by Fuentes et al. ${ }^{[1]}$ but it was higher than a rate of $0.5 \%$ that was reported by Chapron et al. ${ }^{[6]}$ This was probably due to the increased complexity of the cases in the present study compared with that of Chapron et al. ${ }^{[6]}$ which included all laparoscopic gynaecological procedures. Previous studies have shown that complications are greater with increasing complexity of the procedure and pathology. ${ }^{[6,7]}$ Tarik and Fehmi ${ }^{[7]}$ demonstrated that when operations were classified as diagnostic laparoscopy, minor, major and advanced laparoscopic procedures, major complication rates were $1.81 \%, 1.37 \%, 1.82 \%$ and $4.82 \%$, respectively. A study by Johnston et al. ${ }^{[8]}$ showed that all major complications occurred in advanced or major laparoscopic procedures. Moreover, a study conducted by Kafulafula et al. ${ }^{[9]}$ in Durban found a complication rate of $14.8 \%$ for all gynaecological laparoscopies. This high rate was attributed to postoperative pyrexia. ${ }^{[9]}$

\section{Entry-related injury}

Less than a tenth of patients $(1.1 \% ; n=5)$ had entry-related injuries in our present study and occurred during insertion of the primary and secondary port trocars. Secondary trocar insertion was associated with minor bleeding from the inferior epigastric vessels in four cases, while one patient required re-laparoscopy for bleeding and a peritoneal bleeder was identified at the site of primary trocar insertion at the umbilicus. There were no major vascular injuries.
A retrospective review on laparoscopic entry showed that over $70 \%$ of entry-related injuries were vascular and that $80 \%$ of access-related deaths were vascular with aortic and inferior vena cava injuries. ${ }^{[10]}$ Bhoyrul et al. ${ }^{[11]}$ reported that $64.9 \%$ of trocar injuries were vascular and were associated with more fatalities. There were no major vascular injuries and deaths in the present study, and this may be attributed to the fact that we used high intra-peritoneal pressures $(20 \mathrm{mmHg})$ in all of our procedures.

There were no bladder or intestinal injuries with the primary and secondary trocar entry, and this was probably due to careful patient selection and site of port placement. A study by Tarik and $\mathrm{Femi}^{[7]}$ found an injury rate of $0.7 \%$ when Veress and trocar-related entries were combined. It is well known that adhesion formation in the umbilical area is highest in patients with previous midline laparotomy and predisposes patients to injury during laparoscopic entry ${ }^{[10]}$ Entry through the Palmer's point has been advocated for in these patients as it is associated with reduced bowel-related entry injury. ${ }^{[4]}$ This was the approach adopted in our unit and is probably the reason why there was no entry-related bowel injury. The Palmer point entry was used in $17 \%$ of patients in the present study. In additon, all the laparoscopic adnexal surgery in mid-trimester of pregnancy and laparoscopic urogenital fistula repair utilised the Palmer point entry site.

No patient had a Veress needle injury. Veress needle entry is associated with an injury rate of $0.1 \%$, with vascular injury accounting for about half of all patients, bowel injury for $39 \%$ of cases and bladder injury for $6 \% \cdot{ }^{[12]}$ In our unit, we follow standard entry protocols for Veress needle insertion and this may contribute to our good results.

\section{Intraoperative injury}

Less than a tenth of patients $(2.7 \% ; n=12)$ had intraoperative injuries. This was similar to a reported rate of $2.6 \%$ in a retrospective review of complications following laparoscopic surgery performed by a single surgeon in Canada. ${ }^{[13]} \mathrm{A}$ lower rate of $0.5-1.1 \%$ was recorded in two other studies, ${ }^{[5,8]}$ but these studies included both diagnostic and advanced laparoscopic procedures. Bowel injury $(n=6)$ was the most common intraoperative injury in our study, accounting for $50 \%$ of all cases. Similar findings were reported in audits conducted by Johnston et al. ${ }^{[8]}$ and Chapron et al. ${ }^{[6]}$ Shastri et al. ${ }^{[5]}$ and Fuentes et al. ${ }^{[1]}$ reported haemorrhage as the most common intraoperative complication in their studies. All the injuries in our study except one were caused by instrumentation during dissection. One patient presented with delayed symptoms suggestive of a ureteric injury which may have been caused by thermal injury. In a review of intestinal injuries reported by Chapron et al, , ${ }^{[6,14]} 26 / 32$ intraoperative injuries were due to dissection and the other six by electrocautery.

There were two small intestine injuries, three sigmoid colon injuries and one rectal injury, giving an incidence rate of $1.3 \%$. This rate was higher than the $0.03-0.39 \%$ rate reported in a systematic review by Llarena et al. ${ }^{[15]}$ Complexity of our procedure may account for the higher injury rate. The use of laparoscopic scissors during dissection was the cause of all the bowel injuries similar to Chapron et al. ${ }^{[14]}$ However, Llarena et al. ${ }^{[15]}$ reported that $29 \%$ of bowel injuries were due to electrocautery and $15.1 \%$ occurred during dissection of adhesions with scissors. ${ }^{[15]}$ In the present study, all intestinal injuries were identified intraoperatively and repaired. Other studies reported that $\sim$ one-third of the cases were identified intraoperatively with the majority presenting in the postoperative period. ${ }^{[6,14]}$ Injuries 
caused by electrocautery may account for the late discovery. Half of the patients $(50 \% ; n=3)$ had laparoscopic repair, and repair is possible if injury is identified immediately and if minimal faecal soiling occurred, as was done in the two cases with small bowel injury and one case with sigmoid colon injury. A systematic review by Llarena et al. ${ }^{[15]}$ showed that only $8 \%$ of injuries were repaired laparoscopically with the majority requiring conversion to laparotomy. The rectal and sigmoid injury during surgery for endometriosis as well as the small intestine injury during extensive adhesiolysis required conversion to laparotomy for effective repair.

Urological injuries were also seen in four patients. Two patients had bladder injuries $(0.4 \%)$ while the other two had ureteric injuries $(0.4 \%)$. Both bladder injuries occurred during LSCP in patients who had previous anterior repairs. These injuries were identified intraoperatively and corrected laparoscopically. Both patients had extensive adhesions and difficulties were encountered when reflecting the bladder from the vaginal wall. A study addressing only LSCP reported a bladder injury rate of $4.7 \%,{ }^{[16]}$ which is similar to the injury rate in our study if only LSCP was reviewed (5.5\%; $n=36)$. A recognised risk factor for bladder injuries during laparoscopy is adhesions from previous surgery in the region of the bladder, vagina and cervix. ${ }^{[5]}$ This is highlighted in a study by Shastri et al., ${ }^{[5]}$ where five bladder injuries were identified in 335 patients who had laparoscopic hysterectomies. All injuries were in patients who had previous caesarean sections. Bladder injury rates of $0.13-1.5 \%$ have been reported in other studies, ${ }^{[1,5,8]}$ which is comparable with our study.

Specific to the ureteric injuries $(0.4 \%)$, one was identified intraoperatively and the other was suspected after 11 days when the patient presented with non-specific symptoms and deranged renal function tests. Early recognition allowed immediate laparoscopic repair with sutures and cystoscopic stenting while the late case underwent a laparotomy and ureteric implant after 18 days for repair. Most ureteric injuries are diagnosed postoperatively and have been shown to occur less than bladder injuries. ${ }^{[8,17]}$ Our injury rate was comparable to the $0.42 \%$ reported in a study by Tamussino et al., ${ }^{[18]}$ and were lower than the $1.1 \%$ reported by Park et al. ${ }^{[17]}$ Ureteric injuries are common in patients with risk factors such as previous surgery, pelvic inflammatory disease and endometriosis. ${ }^{[17]}$ In our study, both ureteric injuries occurred in patients who had surgery for severe endometriosis, similar to the review by Johnston et al. ${ }^{\left[{ }^{[8]}\right.}$ which found that ureteric injuries occured only in patients who had surgery for endometriosis.

Vascular injuries occurred in two patients $(17 \%$ of all intraoperative injuries). Both injuries were minor. One involved an omental vessel in a patient who had extensive adhesiolysis and the other occurred from surface bleeding in a pedunculated fibroid in a patient who had adnexal surgery in the mid-trimester of pregnancy. Both were repaired laparoscopically without requiring blood transfusion. In a total of 18061 patients who had laparoscopic surgery for advanced or major gynaecological condition, Chapron et al. ${ }^{[6]}$ reported 35 patients with haemorrhage $(0.2 \%)$, of whom 5 had injury to major blood vessels (4 with inferior vena cava and 1 with ileac vessel), while Shastri et al. ${ }^{[5]}$ reported 30 patients with haemorrhage $(0.8 \%)$, of whom 14 required conversion to laparotomy, and these complication rates are comparable to our rate of $0.4 \%$.

Surgery for severe endometriosis accounted for $42 \%(n=5 / 12)$ of intraoperative injuries. These included two ureteric, two sigmoid colon and one rectal injury. A total of 133 patients had surgery for severe endometriosis and the complication rate was $3.6 \%$. A study evaluating complications after surgery for deep infiltrating endometriosis recorded a rate of $2.1 \%{ }^{[19]}$ The need for complete excision of the disease may be a factor associated with injury during surgery. In the audit conducted by Chapron et al. ${ }^{[6]}$ patients who had undergone major adhesiolysis and laparoscopic hysterectomies had the most laparoscopic complications, ${ }^{[6]}$ while the study by Shastri et al. ${ }^{[5]}$ reported that all bowel, bladder and ureteric injuries occurred during total laparoscopic hysterectomy.

\section{Conversion to laparotomy}

Is conversion to laparotomy a complication or good judgement? Some authors have included conversion to laparotomy as a complication, ${ }^{[5]}$ while others have regarded it as good judgement. The surgeon in the present study believes that conversion conducted timeously with good surgical outcome and no morbidity is good judgement and not a complication.

The conversion rate to laparotomy in our present study was $2.5 \%$. In the five conversions to laparotomy due to technical difficulties, adhesions were the common denominator. In a study by Johnston et al. ${ }^{[8]}$ four of the six patients converted to laparotomy were due to technical difficulty from dense adhesions, large fibroid and severe endometriosis. Similarly, in a study by Shastri et al., ${ }^{[5]} 32$ patients were converted to laparotomy, of whom 8 were due to severe adhesions. The other reasons for conversion were as follows: 14 due to haemorrhage; 5 due to bladder injuries; 2 due to ureteric injuries; and 3 due to bowel injuries. ${ }^{[5]}$ Our conversion rate $(1.1 \%)$ was higher than the rate of $0.33 \%$ reported by Johnston et al..$^{[8]}$ but similar to the rate of $1.3 \%$ reported by Shastri et al. ${ }^{[5]}$ However, only advanced procedures were included in the study by Shastri et al. ${ }^{[5]}$ while the study by Johnston et al. ${ }^{[8]}$ included all procedures.

Equipment failure was a non-functioning suction/irrigation apparatus in one case and the absent device was the non-availability of a lapro-bag in the other patient who had surgery for extra-uterine pregnancy (14 weeks). Surgery was completed successfully for the patient with an extra-uterine pregnancy and a small $3 \mathrm{~cm}$ incision was made to remove the fetus.

Three patients had conversion to laparotomy due to intestinal injuries involving the rectum, sigmoid colon and small intestine. All were successfully repaired with laparotomy. Conversions were due to faecal soiling in the sigmoid colon injury, while the small intestinal injury occurred during extensive adhesiolysis and enterolysis, and a decision was made to convert to laparotomy for the entire small bowel to be carefully examined. The rectal injury was converted by a general surgeon who felt that the repair would be better by open surgery. Johnston et al. ${ }^{[8]}$ converted two of six patients due to intraoperative injuries. ${ }^{[8]}$ We had a conversion rate of $0.7 \%$ due to viscus injury and this is comparable with the rate of $0.5-1.6 \%$ that has been reported in the literature. ${ }^{[1,5,8]}$ None of the cases in our study required conversion to laparotomy because of uncontrollable bleeding, although haemorrhage was suspected in one patient. Shastri et al ${ }^{[5]}$ identified uncontrolled intra-abdominal haemorrhage as the most common reason for conversion to laparotomy.

The case of suspected haemorrhage occurred in a patient who had a heterotrophic pregnancy at 12 weeks' gestation. The patient had presented with rupture of a tubal pregnancy. This patient became hypotensive at laparoscopy, although no obvious bleeding vessel could be identified. The anaesthetist was uncomfortable 
with the low blood pressures and because of the large volume of clots in the peritoneum, the upper abdomen could not be explored and conversion to laparotomy was required. With release of the pneumoperitoneum and correction of the Trendelenburg position, the blood pressure normalised. Following evacuation of the clots, no specific vascular bleeder was identified. It was postulated that the pneumoperitoneum may have reduced the venous return to the heart and accounted for the hypotension.

\section{Postoperative complications}

Two patients who had undergone LAVH developed postoperative ileus. Both cases had adhesiolysis and enterolysis performed and the ileus was managed conservatively with drips and nasogastric tubes. Our postoperative complication rate of $0.4 \%$ is comparable to that reported by Shastri et al. ${ }^{[5]}$ The 13 cases identified by Shastri et al. ${ }^{[5]}$ included surgical emphysema, deep vein thrombosis, pulmonary oedema, port site hernia and wound dehiscence. A previous study conducted in Durban, SA, reported postoperative pyrexia as the most common postoperative complication, ${ }^{[9]}$ which was not the case in the present study.

\section{Study limitations}

This was a retrospective study. All the surgical procedures were conducted by a single highly skilled surgeon (SR). Therefore, the true injury rate might have been higher if procedures performed by other less experienced surgeons were included.

\section{Conclusion}

This audit of complex laparoscopic surgery shows that surgical complication rates are low when surgery is performed by a single experienced surgeon and that learning curves reduce adverse surgical events. It has also been shown that the greater the complexity of the procedure, the higher the complication rate. Early identification of injuries either during the procedure or immediately postoperatively leads to decreased morbidity.

Declaration. This study was done in partial fulfilment of requirements of an MMed (O\&G) degree.

Acknowledgements. We would like to thank Dr C Tiloke for assisting with the preparation of the manuscript.
Author contributions. CNO conceptualised the study and wrote the manuscript. SR collected data and wrote the manuscript. JM critically revised the manuscript. All the authors approved the manuscript for publication.

Funding. None.

Conflicts of interest. None.

1. Fuentes MN, Rodriguez-Oliver A, Naveiro Rilo JC, et al. Complications of laparoscopic gynaecologic surgery. JSLS 2014;18(3):e2014.00058. https://doi.org/10.4293\%2FJSLS.2014.00058

2. Wattiez A, Soriano D, Cohen SB, et al. The learning curve of total laparoscopic hysterectomy: Comparative analysis of 1647 cases. J Am Assoc Gynecol Laparosc 2002;9(3):339-345. https:// doi.org/10.1016/s1074-3804(05)60414-8

3. Kirshtein B, Domchik S, Mizrahi S, Lantsberg L. Laparoscopic diagnosis and treatment Kirshtein B, Domchik S, Mizrahi S, Lantsberg L. Laparoscopic diagnosis and treatment
of postoperative complications. Am J Surg 2009;197(1):19-23. https://doi.org/10.1016/j. amjsurg.2007.10.019

4. Kyung MS, Choi JS, Lee JH, Jung US, Lee KW. Laparoscopic management of complications in gynaecologic laparoscopic surgery: A 5-year experience in a single center. J Minim Invasive Gynecol 2008;15(6):689-694. https://doi.org/10.1016/j.jmig.2008.07.006

5. Shastri SS, Singh AA, Darawade SP, Manwani SD. Complications of gynaecologic laparoscopy: An audit. Int J Reprod Contracep Obstetr Gynaecol 2018;7(12):4870-4876. https://doi org/10.18203/2320-1770.ijrcog20184931

6. Chapron C, Querleu D, Bruhat MA, et al. Surgical complications of diagnostic and operative gynaecological laparoscopy: A series of 29966 cases. Hum Reprod 1998;13(4):867-872. https:// doi.org/10.1093/humrep/13.4.867

7. Tarik A, Fehmi C. Complications of gynaecological laparoscopy - a retrospective analysis of 3572 cases from a single institute. J Obstet Gynaecol 2004;24(7):813-816. https://doi. org $/ 10.1080 / 01443610400014857$

8. Johnston K, Rosen D, Cario G, et al. Major complications arising from 1265 operative laparoscopic cases: A prospective review from a single centre. J Minim Invasive Gynecol 2007;14(3):339-344. https://doi.org/10.1016/j.jmig.2006.12.003

9. Kafulafula G, Ramphal S, Moodley J. Complications of gynaecological endoscopic surgery at King Edward VIII Hospital, Durban, South Africa. Trop Doct 2002;32(2):101-102. https://doi org/10.1177/004947550203200219

10. Vilos GA, Ternamian A, Dempster J, Laberge PY, Clinical Practice Gynaecology Committee. Laparoscopic entry: A review of techniques, technologies, and complications. J Obstet Gynaecol Can 2007;29(5):433-447. https://doi.org/10.1016/s1701-2163(16)35496-2

11. Bhoyrul S, Vierra MA, Nezhat CR, Krummel TM, Way LW. Trocar injuries in laparoscopic surgery. J Am Coll Surg 2001;192(6):677-683. https://doi.org/10.1016/s1072-7515(01)00913-9

12. Jansen FW, Kolkman W, Bakkum EA, et al. Complications of laparoscopy: An inquiry about closed- v. open-entry technique. Am J Obstet Gynecol 2004;190(3):634-638. https://doi org/10.1016/j.ajog.2003.09.035

13. Grant-Orser A, El Sugy R, Singh SS. Does laparoscopy safely improve technicity for complex hysterectomy cases? J Obstet Gynaecol Can 2014;36(3):248-252. https://doi.org/10.1016/s17012163(15)30633-2

14. Chapron C, Pierre F, Harchaoui Y, et al. Gastrointestinal injuries during gynaecological laparoscopy. Hum Reprod 1999;14(2):333-337. https://doi.org/10.1093/humrep/14.2.333

15. Llarena NC, Shah AB, Milad MP. Bowel injury in gynaecologic laparoscopy: A systematic review. Obstet Gynaecol 2015;125(6):1407-1417. https://doi.org/10.1097/aog.0000000000000855

16. Coolen AL, van Oudheusden AM, van Eijndhoven HW, et al. A comparison of complications between open abdominal sacrocolpopexy and laparoscopic sacrocolpopexy for the treatment of vault prolapse. Obstet Gynaecol Int 2013;2013:1-7. https://doi.org/10.1155/2013/528636

17. Park JH, Park JW, Song K, Jo MK. Ureteral injury in gynecologic surgery: A 5-year review in a community hospital. Korean J Urol 2012;53(2):120-125. https://doi.org/10.4111/ kju.2012.53.2.120

18. Tamussino KF, Lang PF, Breinl E. Ureteral complications with operative gynaecologic laparoscopy. Am J Obstet Gynaecol 1998;178(5):967-970. https://doi.org/10.1016/s0002-9378(98)70532-0

19. Kondo W, Bourdel N, Tamburro S, et al. Complications after surgery for deeply infiltrating pelvic endometriosis. BJOG 2011;118(3):292-298. https://doi.org/10.1111/j.1471-0528.2010.02774.x

Accepted 2 August 2021. 